TAMÁS ADAMIK

\title{
THEODULFS PALMSONNTAGSHYMNUS
}

\author{
(POETAE LATINI MEDII AEVI L, 558-559 Nr. 69)
}

\begin{abstract}
Summary: By nationality, Theodulf was a Visigoth, born in northern Spain around 750. In 787 he was appointed bishop of Orléans. In 817 Bernard of Italy, nephew of Louis the Pious, revolted against his uncle's rule. Theodulf was implicated in this revolt, therefore Louis banished him to Angers, where Theodulf wrote much of his personal poetry. His style is grounded in a thorough knowledge of Classical Latin, yet imbued with his own distinctive nuances. His Hymn on Palm-Sunday Gloria, laus, et honor mirrors well both the contemporary events and his devotion to classical models and to the Bible.
\end{abstract}

Key words: Theodulf, Hymn on Palm-Sunday Gloria, laus, et honor.

1. Theodulf stammte aus Spanien, vielleicht aus Saragossa, und war westgotischer Abkunft. Er war jünger als Alkuin (etwa 760-821), ${ }^{1}$ und als dieser sich vom Hof Karls des Großen zurückgezogen hatte, wurde Theodulf wichtigster Ratgeber des Kaisers in theologischen und ethischen Fragen. Karl hat ihm mehrere Abteien und das Bistum Orléans gegeben. ${ }^{2}$ Im Jahre 798 wurde er zusammen mit Leidrad von Lyon von Karl zum missus dominicus in der Provence ernannt, und im Jahre 800 nahm er am Romzug teil, das heißt, er stand bei Karl in hohem Ansehen, ${ }^{3}$ und auch bei seinem Nachfolger Ludwig bis 817. In diesem Jahr brach der Aufstand König Bernhards von Italien gegen Ludwig aus. Ludwig besiegte Bernhard, und im Jahre 818 wurde Theodulf als Mitverschwörer Bernhards verhaftet und im Kloster St. Aubin in Angers in Haft gehalten. Theodulf behauptete mehrmals seine Schuldlosigkeit, aber ohne Erfolg. Das von ihm geforderte Schuldbekenntnis lehnte er ab. Er starb in der Verbannung vor dem Oktober 821, als eine allgemeine Amnestie für die Teilnehmer am Aufstand Bernhards erlassen wurde. ${ }^{4}$

Theodulf hat im Auftrage Karls mehrere Prosawerke geschaffen, zum Beispiel De Spiritu Sancto (809) und De ordine baptismi (812). Er hat an der Redaktion der Libri Carolini im Jahre 790 teilgenommen. Er beschäftigte sich mit dem Text der Bibel, das heißt, mit Textkritik und biblischer Philologie. Diese Werke bilden einen Teil der

\footnotetext{
${ }^{1}$ Vgl. Elisabeth DAhlHAUS-BERG, Nova antiquitas et antiqua novitas. Typologische Exegese und isidorianisches Geschichtsbild bei Theodulf von Orléans. Köln, Wien 1975, 5-7.

${ }^{2}$ Vgl. Elisabeth DAHLHAUS-BERG, op. cit. 9-10.

${ }^{3}$ Vgl. Elisabeth DAHLHAUS-BERG, op. cit. 12-15.

${ }^{4}$ Vgl. Elisabeth DAHLHAUS-BERG, op. cit. 16-21.
} 
karolingischen kulturellen und religiösen Erneuerung und haben heute nur noch kulturgeschichtliche Bedeutung. ${ }^{5}$ Seine Poesie ist es, der Theodulf seine Unsterblichkeit verdankt.

In der Fachliteratur betrachtet man Theodulf als den größten Dichter der karolingischen Zeit. M. Manitius konzipiert wie folgt: „Nach alledem haben wir uns in Theodulf einen Mann vorzustellen, dessen schöngeistige Veranlagung ihn zum häufigen Genusse antiker Dichtung und Kunst trieb, und der infolge seiner ästhetischen Bildung wohl die meisten seiner Zeitgenossen übersah. “6 L. Alfonsi nennt ihn ,il maggior poeta di questa prima generazione“" ${ }^{7}$ Fr. Brunhölzl betont Theodulfs poetische Spontaneität und Humanität. ${ }^{8}$ E. R. Curtius schreibt über die Allegorese wie folgt: „Ein Platoniker des 12. Jahrhunderts, Johannes von Salisbury, hat das Grundmotiv der antiken Allegorese rein und elegant formuliert: Entheticus $183 \mathrm{ff}$. ,Dringe darauf, daß die Philologie sich dem Mercur geselle: nicht etwa, damit den falschen Gottheiten Ehrfurcht erwiesen werde; sondern unter der Decke der Worte sind Wahrheiten verborgen. ' ... Dieselbe Theorie in karolingischer Zeit bei Theodulf (Poetae I 543, 19 ff.)." ${ }^{\text {"9 }}$ Die Allegorese bietet eine Möglichkeit für Theodulf, die heidnischen antiken Poeten zu würdigen. Laut P. Klopsch: „Die Anschauung, daß unter Verhüllung des Wortsinnes eine tiefere Wahrheit zu finden sei, ist nicht nur bezüglich Vergils geäußert worden; schon Theodulf von Orléans hat Ovid neben ihn gestellt (Carm. 45, 19 f.; MGH Poet. 1, 543):

\section{In quorum dictis quamquam sint frivola multa, plurima sub falso tegmine vera latent. “10}

L. Nees hebt die Harmonie der verbalen und visuellen Materialien bei Theodulf hervor. ${ }^{11}$ J. Pucci betont, daß Theodulfs Stil auf dem klassischen Latein beruht, doch von persönlichem Gefühl durchtränkt ist. ${ }^{12}$

E. Duemmler hat die kritische Ausgabe der Gedichte von Theodulf verfertigt. ${ }^{13}$ In dieser Ausgabe sind 79 Gedichte zu finden, insgesamt erhalten sind über 4600 Verse, zumeist elegische Distichen. Charakteristisch ist die starke Beeinflussung durch Ovid und Prudentius. Die von ihm gepflegten Gattungen sind Briefgedicht,

\footnotetext{
${ }^{5}$ Über diese Werke s. Elisabeth DAHLHAUS-BERG, op. cit. 21-235.

${ }^{6}$ Max MANiTIUS, Geschichte der lateinischen Literatur des Mittelalters. Erster Teil: Von Justinian bis zur Mitte des zehnten Jahrhunderts. München 1911, 538.

${ }^{7}$ Luigi ALFONSI, La letteratura latina medievale. Firenze, Milano 1972, 76.

${ }^{8}$ Franz BRUNHÖLZL, Histoire de la littérature latine du moyen age. I/2 L'époque carolingienne. Brepols 1991, 51.

${ }_{9}^{9}$ Ernst Robert CURTIUS, Europäische Literatur und latenisches Mittelalter. Bern 1958, 211 Anm. 1. 1980, 105

${ }^{10}$ Paul KLOPSCH, Einführung in die Dichtungslehren des latenischen Mittelalters. Darmstadt

${ }^{11}$ L. NEES: A Tainted Mantle: Hercules and the Classical Tradition at Carolingian Court. Philadelphia 1991

${ }^{12}$ Medieval Latin. Second Edition. Edited by K. P. HARrington. Revised by Joseph PuccI. With a grammatical introduction by Alison Goddard ELLIOTT. Chicago and London 1997, 233.

${ }^{13}$ MGH Poetae Latini aevi Carolini. Recensuit Ernestus DUEMMLER. Tomus I. Unveränderter Nachdruck der Ausgabe von 1881. München 1978, 437-581.
} 
theologisch-moralisches Lehrgedicht (Hymnus ${ }^{14}$ ) und Inschrift. D. Schaller hat Dümmlers Ausgabe scharf kritisiert und zu mehreren Gedichten Theodulfs kritische Bemerkungen gemacht, aber den Palmsonntagshymnus außer acht gelassen: „Vom Palmsonntagshymnus Theodulfs (Nr. 69), dessen reiche liturgische Überlieferung erst die Ausgabe in den ,Analecta hymnica' (Bd. 50) herangezogen hat, können wir hier absehen." 15

J. Szövérffy hat grundlegende Feststellungen über den Palmsonntagshymnus Theodulfs Gloria, laus et honor gemacht. Der originelle Text hat 39 Distichen, deren erste sechs bei der Prozession gesungen wurden und bis heute in der Liturgie verwendet werden. Das erste Distichon dient als Refrain: es wird nach jedem folgenden wiederholt. Laut Hugo von Fleury (MGH SS 9, 363-64) ist der Verfasser Theodulf von Orléans, der den Hymnus als Gefangener in Angers beim Vollzug der Palmsonntagsprozession gedichtet hat. Als Ludwig der Fromme vor dem Turm, in dem Theodulf in Haft gehalten wurde, in der Prozession stehenblieb, hörte er Theodulf diesen Hymnus singen. Ludwig wurde gerührt und verzieh ihm. Der Text hat biblischen Hintergrund (Matth. 21, 1-3, 8-11), der Einzug von Christus in Jerusalem wird in mystisch-allegorischem Sinne interpretiert. Seine Distichen hat nicht nur die klassische Tradition, sondern auch das Auferstehungsgedicht Tempora florigero von Venantius Fortunatus beeinflußt. ${ }^{16}$ Laut F. J. E. Raby ist der Palmsonntagshymnus das beste Gedicht von Theodulf: es ist die Krone seiner Dichtung. ${ }^{17}$ Trotz solcher Meinungen hat meines Wissens noch niemand dieses Gedicht eingehender analysiert. Mit meinem Aufsatz möchte ich diesem Mangel abhelfen.

2. Zunächst zur Struktur und zum Wortbestand des Hymnus! Wie gesagt, besteht der vollständige Text des Gedichtes aus 39 Distichen beziehungsweise aus 78 Versen. Dieses lange Gedicht zerfällt in zwei fast gleiche Teile. Der erste Teil umfaßt Vers 1-38, der zweite Vers: 39-78. Die Separation der zwei Teile ist so markant, daß H. Spitzmuller in seiner Anthologie nur den ersten Teil publiziert hat. ${ }^{18}$ Obwohl er seine Entscheidung nicht begründet, fühlt man, daß er den ersten Teil für das Wesen des Gedichtes gehalten hat. Im ersten Teil findet die mystisch-allegorische Interpretation von Jesu Einzug in Jerusalem statt; der zweite Teil besteht aus einer genauen Aufzählung der Teilnehmer der Palmsonntagsprozession in Angers.

Betrachten wir zuerst die Struktur des ersten Teiles. Das erste berühmte Distichon ist eine Akklamation des Königs Christus und zugleich die Ankündigung, daß ein Kinderchor diesen Hymnus in der Palmsonntagsprozession singt:

\footnotetext{
${ }^{14}$ Unter Theodulfs Namen stehen vier Hymnen in den $A H$; vgl. Joseph SzÖVÉRFFY: Annalen der lateinischen Hymnendichtung I: Die lateinischen Hymnen vom Ende des des 11. Jahrhunderts. Berlin 1964, 202. (1962).

${ }^{15}$ D. SCHALLER: Philologische Untersuchungen zu den Gedichten Theodulfs von Orléans: DA 18

${ }^{16}$ Joseph SZÖVÉRFFY, „Gloria, laus, et honor“, New Catholic Encyclopedia VI (1967) 511-512.

${ }^{17}$ F. J. E. RABY, A History of Christian-Latin Poetry. From the beginnings to the close of The middle ages. Second Edition. Oxford 1953, 175.

${ }^{18}$ Poésie latine chrétienne du moyen age, IIIe-XVe siècle. Textes recueillis, traduits et commentés par Henry SPITZMULLER. Desclée de Brouwer, Bruges 1971, 260-263.
} 


\section{Gloria laus et honor tibi sit, rex Christe redemptor. \\ Cui puerile decus prompsit osanna pium (1-2).}

Dann folgt der erste Abschnitt (3-6), der auch in den klassischen heidnischen Hymnen obligatorisch ist: Darlegung der Herkunft und des Wirkungskreises der Gottheit:

Israhel es tu rex, Davidis et inclita proles,

Nomine qui in domini, rex benedicte, venis.

Coetus in excelsis te laudat caelicus omnis,

Et mortalis homo et cuncta creata simul (3-6).

Schon diese sechs Zeilen illustrieren schön, daß Theodulf sprachlich und inhaltlich die klassische Tradition mit der christlichen in Einklang gebracht hat. Alle Worte des ersten Distichons - außer osanna - sind klassisch. Die drei ersten Worte: gloria, laus et honor sind die klassischen Termini des Ruhmes und der Lobpreisung. Als Cicero über die Ruhmsucht der Sportler schreibt, gebraucht er die Worte: gloria, honestas, laus, dignitas, decus (Tusc. 1, 46). Aeneas lobt Dido wie folgt: semper honos nomenque tuum laudesque manebunt (Aen. 1, 909). Vgl. Tert. Adv. Val. 12 p. 191, 19: in honorem et gloriam patris; Vulg. Deut. 26, 19: gentes, quas creavit in laudem et nomen et gloriam suam. Die Wortfügung rex Christe entstammt aus den Evangelien: Ecce rex venit tibi mansuetus ${ }^{19}$ (Matth. 21, 5); rex Israel, ... ecce rex tuus venit (Joh. 12, 13, 15). Bezüglich des Wortes rex schreibt J. Szövérffy wie folgt: „Besonders interessant ist auch die Hervorhebung des Königs-Charakters Christi. “" ${ }^{20}$ Aber wenn man in Betracht zieht, daß Theodulf die Beschreibung der Evangelien treu wiedergibt, ist es nur natürlich, daß er den König-Charakter Christi betont. Die wunderschöne Wortfügung puerile decus nimmt Theodulf von Statius: hoc puerile decus (Achill. 2, 119) und setzt sie an dieselbe Stelle des Verses. Die Wortfügung prompsit osanna ist klassisch und christlich zugleich. Das Verb promo ist in dieser Bedeutung bei Tacitus zu finden: magnificam orationem de semet ipso prompsit (Hist. 2, 90). Das Wort osanna entnimmt Theodulf aus den Evangelien: turbae autem, quae praecedebant, et quae sequebantur, clamabant, dicentes: Hosanna filio David: benedictus qui venit in nomine Domini: hosanna in altissimis (Matth. 21, 9); In crastinum autem turba multa, quae venerat ad diem festum, cum audissent quia venit Iesus Ierosolymam, acceperunt ramos palmarum, et processerunt obviam ei, et clamabant: Hosanna, benedictus qui venit in nomine Domini, rex Israel (Joh. 12, 12). Aber man kann auch mit Iuvencus' Einfluß rechnen; vgl. Iuvenc. 3, 641.

Die Wortfügungen der dritten Zeile Israel ... rex, Davidis proles stammen aus Johannes beziehungsweise aus Matthäus: rex Israel (Joh. 12, 13); filio David (Matth. 21, 9). Diese letzte Stelle weist darauf hin, daß Theodulf zu seinem Hymnus das

\footnotetext{
${ }^{19}$ Den lateinischen Text der Bibel zitiere ich aufgrund der Ausgabe: Biblia Sacra iuxta Vulgatam Clementinam. Nova editio logicis partitionibus aliisque subsidiis ornata a Alberto COLUNGA, O. P. et Laurentio TURRANDO. Matriti 1994.

${ }^{20}$ Joseph SzÖVÉRFFY, Annalen der lateinischen Hymnendichtung I: Die lateinischen Hymnen bis zum Ende des 11. Jahrhunderts. Berlin 1964, 203.
} 
Evangelium von Matthäus ${ }^{21}$ ebenso gebraucht hat wie das des Johannes. Die Wortfügung inclita proles ist klassisch und poetisch; vgl. inclute Memmi (Lucr. 5, 8); dux inclute Teucrum (Verg. Aen. 6, 562); prolemque propagant (Lucr. 2, 997); laudantur simili prole puerperae (Hor. Carm. 4, 5, 23). Die 4. Zeile stammt aus den Evangelien. Die Wortfügung coetus ... caelicus ist klassisch: o dulces comitum valete coetus (Catull. 49, 6); virgineos ... coetus deserere (Ov. Fast. 2, 173); in senatu caelico (Mart. Cap. 9, 891). Der Ausdruck in excelsis ist ekklesiastisch; vgl. Gloria in excelsis deo. Die attributive Konstruktion mortalis homo ist klassisch; vgl. homo ad immortalium cognitionem nimis mortalis est (Sen. Dial. 8, 5, 7). Die Wortfügung cuncta creata stammt aus Venantius Fortunatus: dantque creatori cuncta creata precem (MGH 4, 1; 3, 9, 38), und Theodulf setzt sie an dieselbe Stelle der Verszeile.

Hierauf folgt der zweite Abschnitt des ersten Teiles (7-20), in dem Theodulf die Teilnehmer der Prozession mit dem jüdischen Volk in Parallele stellt. Diese fortwährend wiederkehrende Parallele bildet den Rahmen der moralischen Interpretatio: jedes Distichon enthält eine Gleichsetzung:

Plebs Hebraea tibi cum palmis obvia venit: Cum prece, voto, hymnis adsumus ecce tibi (7-8).

Theodulf spricht zu Jesus im Namen der Gemeinschaft, darum sagt er immer „wir“ (nos):

Hi tibi passuro solvebant munia laudis,

Nos tibi regnanti pangimus ecce melos (9-10).

Das hebräische Volk lobt den bald leidenden Christus, die Bewohner von Angers loben den herrschenden Christus.

Besonders interessant ist die Hervorhebung der vornehmen Herkunft des hebräischen Volkes:

Fecerat Hebraeos hos gloria sanguinis alti:

Nos facit Hebraeos transitus ecce pius (13-14).

Jene sind Hebräer durch ihre vornehme Herkunft, wir durch den frommen „Übergang“'. In Prudentius' Apotheosis fungiert das jüdische Volk als sündhaft; vgl. 421 ff. Dagegen sind die Hebräer bei Theodulf wahre Christen, die Christen aber wahre Hebräer. Diese Anschauung Theodulfs können wir mit dem Einfluß des Apostels Paulus erklären, der schreibt: „Herrlichkeit, Ehre und Friede werden jedem zuteil, der das Gute tut, zuerst dem Juden“"22 (gloria autem, et honor, et pax omni operanti bonum, Iudaeo primum, Röm. 2, 10). Noch stärker: „Jude ist nicht, wer es nach außen ist, und Beschneidung ist nicht, was sichtbar am Fleisch geschieht, sondern Jude ist, wer es im Verborgenen ist, und Beschneidung ist, was am Herzen durch den Geist, nicht durch den Buchstaben geschieht“" (Röm. 2, 28-29). Das Wort transitus

\footnotetext{
${ }^{21}$ Nach Joseph Szövérffy hat Theodulf Matthäus (New Catholic Encyclopedia 511-512), nach Henry Spitzmuller Johannes (op. cit. p. 357. Anm. 19) gebraucht.

${ }^{22}$ Den deutschen Text der Bibel zitiere ich von der Ausgabe: Die Bibel. Einheitsübersetzung. Altes und Neues Testament. Herder, Freiburg-Basel-Wien 1996.
} 
(Übergang) bedeutet hier nicht nur die Prozession, sondern auch die Veränderung, die Bessergestaltung des Lebens. Dies geht klar aus dem folgenden Distichon hervor:

Inclita terrenis transitur ad aethera victis,

Virtus et a vitiis nos capit alma tetris (15-16).

Die Veränderung verläuft von der Erde zum Himmel, von der Schuld zur Tugend. Hier können wir mit dem Einfluß von Ambrosius rechnen, der Pascha als transitus interpretiert: Siquidem verno tempore filii Israel Aegyptum reliquerunt, et per mare transierunt, baptizati in nube, et in mari, ut Apostolus dixit (1 Cor. X, 1 et seq.): et eo tempore Domini quotannis Jesu Christi Pascha celebratur, hoc est, animarum transitus a vitiis ad virtutem, a passionibus carnis ad gratiam sobrietatemque mentis, a malitiae nequitiaeque fermento ad veritatem et sinceritatem (Hexaem. $1,4,14) .{ }^{23}$ Der Dichter weiß, daß dieser Übergang nicht leicht ist, weil der Mensch immer sündhaft bleibt. Er bittet Jesus, diese Sündhaftigkeit als Leichtfertigkeit der Kinder zu betrachten und uns zu helfen, durch Tugend alt zu werden, damit wir den Weg unserer vornehmen Vorfahren finden können:

Nequitia simus pueri, virtute vieti,

Quod tenuere patres, da ut teneamus iter (17-18).

Auch hier können wir auch mit dem Einfluß des Apostels Paulus rechnen, der schreibt: Fratres, nolite pueri effici sensibus, sed malitia parvuli estote: sensibus autem perfecti estote (1 Kor. 14, 20); ut iam non simus parvuli fluctuantes, et circumferamur omni vento doctrinae in nequitia hominum (Eph. 4,14).

Auch in diesem Abschnitt hat Theodulf viel aus der klassischen römischen Literatur geschöpft; zum Beispiel: solvebant munia (9) - omnia solvisti (Verg. Aen. 6, 510); pangimus ... melos (10) - tibi Musarum pangit melos (Enn. Ann. 299); gloria sanguinis alti (13) - quid prodest ... longo sanguine censeri (Iuv. 8, 2); transitus (14) - nam brevis in gelidas membris exilibus undas / transitus est (Ov. Met. 5, 434); transitur ad aethera (15) - vitium ... in lumina mentis transit (Ov. Met. 1, 68); virtus ... alma (16) - almae virtutis (Val. Flacc. 1, 850); a vitiis ... taetris (16) - nullum vitium taetrius est (Cic. Off. 2, 77); nequitia simus pueri (17) - nequitia est, quae te non sinit esse senem (Ov. Trist. 1, 414'); virtute vieti (17) - exile ... et vietum cor fuisse (Cic. Div. 2 , 37); ut ... marcida demittant (lilia) ... caput vietum (Ov. Met. 10, 192); da ut teneamus iter (18) - mediae ... teneamus urbis iter (Verg. Aen. 2, 360); degeneresque ... ne simus ... ab arte (19) - iuvenis patrii non degener oris (Ov. Pont. 3, 5, 7); a domestico ... sermone degeneres (Curt. 7, 5, 29); post illos gratia sancta trahat (20) - trahit sua quemque voluptas (Verg. Ecl. 2, 65); trahunt promissa puellas (Ov. Ars 1, 631). Hier kann man auch mit dem Einfluß des Hohen Lieds rechnen: Trahe me, post te curremus (3).

Im dritten Abschnitt des ersten Teiles (21-38) folgt die anagogische Interpretation: Jesus sei jener, der sich auf den Esel setzt, wir seien der Esel, damit die Stadt Gottes uns zusammen mit Jesus aufnehmen könne:

Sis pius ascensor, tuus et nos simus asellus, Tecum nos capiat urbs veneranda dei (21-22).

${ }^{23}$ Vgl. Christine MohrmanN, „Pascha, passio, transitus“. Ephemerides Liturgicae 66 (1952) 37-52. 
In diesem Teil folgt Theodulf der Beschreibung des Matthäus: Et adduxerunt asinam, et pullum: et imposuerunt super eos vestimenta sua, et eum desuper sedere fecerunt $(21,7)$. Theodulf bittet um ein apostolisches Kleid, das heißt, um den wahren Glauben für die Teilnehmer der Prozession, damit sie Jesus tragen können:

Vestis apostolicae rutilo fulgore tegamur,

Te bene tecta ut ea nostra caterva vehat (23-24).

Matthäus setzt wie folgt fort: Plurima autem turba straverunt vestimenta sua in via: alii autem caedebant ramos de arboribus, et sternebant in via $(21,8)$. Theodulf interpretiert alle Elemente dieser Beschreibung in mystischem Sinn: die hingebreiteten Kleider sind unser Leib und unsere Seele, die Palmenzweige - unser Sieg über das Schlechte, die Weidenzweige - unser reines Herz, die Ölzweige - unsere Sanftmut, die Stadt - der Himmel:

Tegmina sive animas sternamus corpora nostra,

Quo per nos semper sit via tuta tibi.

Sit pia pro palmae nobis victoria ramis,

Ut tibi victrici sorte canamus ita (25-28).

Wenn wir in Prozession in die Stadt einziehen, bedeutet es in anagogischem Sinne, daß wir in den Himmel kommen:

Urbem ut cum ramis et laudibus imus ad istam,

Celsa poli meritis fac ita adire piis (37-38).

Auch in diesem Abschnitt sind Worte und Wortfügungen klassisch: sie sind bei den klassischen römischen Autoren oder bei den spätrömischen christlichen Schriftstellern zu finden; zum Beispiel: ascensor (21) - ascensor vir bigae equitum (Vulg. Is. 21, 9); asellus (21) - demitto auriculas, ut iniquae mentis asellus (Hor. S. $1,9,20)$; urbs veneranda dei (22) - hier assoziiert Theodulf auch Rom, weil das Substantiv urbs auch an sich selbst Rom bedeuten kann; vgl. sine magistratibus urbem esse (Cic. Att. 7, 11, 4); das ist die Ursache, warum er Angers urbs nennt: Quos habet Andegavis venerabilis ambitus urbis (43); rutilo fulgore (23) - fulgor ... rutilus ... quem Martium dicitis (Cic. Rep. 6, 17); nostra caterva (24) - cum magna caterva togatorum (Cic. S. Rosc. 135); tegmina (25) - mihi amictui est Scythicum tegmen (Cic. Tusc. 5, 90); castaque ... praecordia (29) - mollia cinguntur tenui praecordia libro (Ov. Met. 1, 549); prata ad amoena (30) - loca amoena (Sall. Cat. 11, 5); devenere ... amoena virecta fortunatorum nemorum (Verg. Aen. 6, 638); viror (30)pratorum virores (Apul. Fl. 10); annua festa (36) - Festa piae Cereris celebrabant annua matres (Ov. Met. 10, 431); celsa poli (38) - celsi ... dominator poli (Sen. Ep. 107, 10); labere, nympha, polo (Verg. Aen. 11, 588).

Der zweite Teil hat dieselbe Struktur wie der erste Teil: er beginnt mit einer Anrede, dann folgen drei größere Abschnitte.

In der Anrede bittet Theodulf Jesus um Hilfe für die Teilnehmer der Prozession in Angers: 
Hanc in amore tuo collectam respice plebem.

Suscipe et illius vota precesque libens (39-40).

Im ersten Abschnitt (41-48) betont der Dichter, daß alle Bewohner der Stadt - die Priester, das einfache Volk, Männer und Frauen - den Ruhm Jesu singen, darum lobt er die Stadt Angers:

Ecce sacerdotum turbae, popularis et ala, Concinit et laudes sexus uterque tibi.

Quos habet Andegavis venerabilis ambitus urbis, Qui pia devota carmina mente canunt.

Fruge, ope, nundinis, pulchris et rebus abundans, Obsita seu sanctis est bene tota locis (41-44; 47-48).

Im zweiten Abschnitt (49-68) zählt er die heiligen Orte auf. Jeder Ort bekommt ein Distichon. Das Ziel der Aufzählung ist zu zeigen, wie viele heilige Orte und Heilige in Angers sind. Er führt alle Heiligen von Angers und Gallien an: Albinus, Martinus, Saturninus, Sergius, Maurilus, Anianus, Germanus und deren Nachfolger und Nachfolgerinnen:

Plebs venit Albini sancta haec de sede beati, Cum voto et ramis carmina digna ferens.

Illaque baptistae concurrit ab arce Iohannis, Consocians ramis dicta canora suis.

Nec, Martine, cohors tua serior, alme, recurrit, Fertque manu ramos, mente et ore melos.

Turba Saturnini venit his sociata beati, Quam crux et rami, laus et adornat ovans (49-56).

Die Menge trägt Zweige, singt und jubelt, wie der Dichter in allen Pentametern hervorhebt. Est ist eine poetische Bravour, diese Variationen über das Thema von ,singen und jubeln' in Pentametern zu verwirklichen, besonders wenn man bedenkt, daß Theodulf diesen Hymnus in Angers im Gefängnis geschrieben hat.

Im dritten und letzten Abschnitt (69-78) beschreibt Theodulf das Ende der Prozession: Die Menge trifft den Bischof der Michaels-Kirche, und sie preisen zusammen Gott. Von da ziehen sie zur Kathedrale des heiligen Moritz, die - wie eine Mutter - die Menge vereinigt. Hier segnet der Bischof die Gläubigen und schickt sie nach Hause: jeden an seinen eigenen Ort - in wörtlichem und übertragenem Sinne:

Nostra ubi nos prex et benedictio praesulis ornet,

Cum laude ac mittat ad sua quemque loca (77-78).

Der Wortgebrauch ist auch im zweiten Teil klassisch; zum Beispiel: respice plebem (39) - di homines respiciunt (Pl. Rud. 1316); suscipe ... vota (40) - vota suscipi dicitis oportere (Cic. N. D. 3, 93); sexus uterque (42)-calciamenta utrique sexui convenientia (Col. 12, 3, 1); ambitus urbis (43) - urbium ambitu (Quint. 11, 2, 21); devota ... mente (44) - mente secunda (Stat. Theb. 1, 285); rate cum laevi (46)in inpiam Aiacis ratem (Hor. Epod. 10, 14); levi cum sanguine (Verg. Aen. 5, 328); 
nundinis (Markt, Handel) (47) - instituere nundinas in agris (Plin. Ep. 5, 4, 1); pulchris et rebus abundans (47) - vir abundans bellicis laudibus (Cic. Off. 1, 78); obsita ... sanctis ... locis (48) - terga cavis super obsita conchis (Ov. Met. 4, 725); concurrit ab arce (51) - concurrere in arcem (Verg. Aen. 2, 315); consocians ramis (52) - consociare amant ramis (Hor. Carm. 2, 3, 10); his sociata (55) - natam sociare Latinis (Verg. Aen. 7, 96); cella tuo vocitata e nomine (57) - quae quondam magna vocitata est (Cic. De orat. 3, 139); mittere gestit (58) - senatum ... delere gestit (Cic. Phil. 4, 14); gesta beata (60) - historia est gesta res (Cic. Inv. 1, 27); salutiferae ... Mariae (65) - da salutiferam iam mihi fratris (i. e. Apollo) opem (Ov. Ep. 20, 174); praesulis eximii pontificisque sui (68) - praesulem sibi non placuisse (Cic. Div. 1, 55); eximiae laudis amore (Verg. Aen. 7, 496); pontifices veteres (Cic. De orat. 3, 73); scandimus ... sanctum ... clivum (69) - dum Capitolium scandet ... pontifex (Hor. Carm. 3, 30, 8-9); dulcis ... amor (70)-dulci ... adfatus amore est (Verg. Aen. 6, 455); a capite et membris (72) - unum hominem caput ... imperii Romani esse (Liv. 38, 51, 4); fidae dulcia membra domus (Ov. Tr. 1, 3, 64); Mauritii ... ad atria (73) - Iovis atria (Ov. Fast. 3, 703); vox sonet (74) - voce inani sonare (Cic. Fin. 2, 48); specimen istius urbis (76) - specimen fuisse humanitatis (Cic. Tusc. 5, 55) usw.

3. Das bisher Gesagte zusammenfassend können wir bezüglich der Struktur des Hymnus feststellen, daß sie in zwei völlig symmetrische Teile zerfällt: beide beginnen mit einer Anrede, dann folgen drei Abschnitte. Im ersten Teil erzählt Theodulf, was die Teilnehmer der Palmsonntagsprozession sagen, im zweiten Teil spricht er darüber, wer die Teilnehmer sind. Mit dieser symmetrischen Struktur des Hymnus weist der Dichter darauf hin, wie wichtig die Stadt Angers und ihre Bewohner für ihn waren. Darum widmet er die Hälfte des Hymnus dem Lob der Stadt und darum zählt er alle heiligen Orte und Heiligen der Stadt auf, darum setzt er auch Angers mit dem himmlischen Jerusalem gleich. Alles in allem genommen wollte Theodulf die Stadt Angers, deren christliche Gemeinschaft und Bischof in ihrer Bedeutung vergrößern, um sich das Wohlwollen der Stadt und ihres Bischofs zu verschaffen. Warum? Die Antwort können wir in den Gedichten 71 (Ad Aiulfum episcopum) und 72 (Incipit epistola Theodulfi episcopi ad Modoinum episcopum scribens ei de exilio) finden. Im 71. Gedicht bittet er um Aiulfus' Hilfe:

Te precor, interea nostrae memor esto ruinae, Oratu et precibus fors mala nostra leves (59-60).

Dasselbe erbittet er vom Bischof Modoinus im 72. Gedicht:

Fessis opto libens certa succurrere rebus,

Et fratrum mentes sollicitato pias (35-36).

Und dasselbe will er auch beim Bischof von Angers mit dem zweiten Teil seines Palmsonntagshymnus erreichen, weil er ,ein rein kirchliches Verfahren von der Bischofssynode “24 verlangt und dazu die Gunst mehrerer Bischöfe erlangen möchte.

Was den Wortbestand des Hymnus betrifft, können wir behaupten, daß Theodulf in der Wortwahl klassisch ist, das heißt, er schöpft die Worte und Wortfügungen sei-

${ }^{24}$ Elisabeth DAHLHAUS-BERG, op. cit. 21. 
nes Hymnus aus der Bibel, aus spätrömischen christlichen und klassischen römischen Autoren. Er verhehlt uns nicht, daß er auch die heidnischen Autoren zu lesen pflegt:

Legimus et crebro gentilia scripta sophorum, Rebus qui in variis eminuere satis $(45,9-10)$.

Er zählt uns die von ihm gelesenen heidnischen Autoren in diesem Gedicht auf:

Et modo Pompeium, modo te, Donate, legebam, Et modo Virgilium, te modo, Naso loquax $(45,17.18)$.

Die von uns erschlossenen klassischen Parallelen beweisen, daß Theodulf nicht nur die Grammatiker Pompeius, Donat und die Dichter Virgil und Ovid gelesen hat, sondern auch viele andere: Plautus, Terenz, Cicero, Sallust, Lukrez, Horaz, Seneca, Curtius Rufus, Columella, Plinius, Valerius Flaccus, Quintilianus, Statius, Tacitus und Apuleius. Die Muttersprache Theodulfs war nicht mehr lateinisch und wenn er im Lateinischen authentisch sein wollte, schrieb er mit Wortverbindungen und Formeln der heidnischen und christlichen römischen Autoren, die noch eine muttersprachliche Kompetenz im lateinischen Sprachgebrauch hatten. Darum würde ich seine übernommenen Sprachformeln nicht ,Spolien ${ }^{25}$ nennen, weil dieses Wort eine negative Konnotation hat.

Die Restauration des korrekten lateinischen Sprachgebrauchs war ein positives Element in der karolingischen Renaissance. ${ }^{26}$ P. Lehmann stellt wie folgt fest: „Die mittelalterlichen Bücherverzeichnisse sind aus dem 8. Jahrhundert sehr spärlich erhalten. In den ältesten Verzeichnissen von Sainte-Vandrille aus der Zeit von etwa 742-800 kommt kein Buch der 7 freien Künste vor (Gottlieb. no. 1033 ff.) außer einer Arithmetik. Alle Codices sonst enthalten geistige Literatur und die Geschichtswerke des Jordanes; die früheste von mir entdeckte Liste in einer Fuldaer Hs. weist von antiken Texten nur einen Liber Alexandri auf, also wohl ein Werk über Alexander den Großen. ${ }^{627}$ Anstelle der spärlichen Bücherverzeichnisse kann - meiner Meinung nach auch der Wortbestand eines mittelalterlichen Autors ein Bild davon geben, welche für klassischen lateinischen Werke er gelesen hat. Theodulf hat mit hoher künstlerischer Begabung vieles von den römischen Klassikern in seine Dichtung übernommen, so wie Einhart in der Prosa, in seiner Vita Karoli Magni, in der er mit Worten und Wortfügungen von Sueton das Leben Karls des Großen beschrieben hat. ${ }^{28}$ Beide wollten bei den klassischen römischen Autoren formale Modelle finden, aber mit den formalen haben sie auch inhaltliche Formen gefunden. Und dies beweist ihre hohe künstlerische Empfänglichkeit und weist auf das Wesen der karolingischen Renaissance hin.

Eötvös-Loránd-Universität

Institut für Klassische Philologie

H-1364 Budapest, Pf. 107

${ }^{25} \mathrm{Vgl}$. Elisabeth DAHLHAUS-BERG, op. cit. 33.

${ }^{26}$ Paul Lehmann, Das Problem der karolingischen Renaissance, in: Erforschung des Mittelalters, Stuttgart 1959, Bd. 2, 109-138.

${ }^{27}$ Paul LeHMANN, op. cit. 115

${ }^{28}$ Paul LehmanN, Das literarische Bild Karls des Großen vornehmlich im lateinischen Schrifttum des Mittelalters. In: Erforschung des Mittelalters, Stuttgart 1959, Bd. 1, 163-166. 